\title{
MTL-A Multi-Wire Transmission Line Modeling Toolbox
}

\author{
Thomas Magesacher
}

\begin{abstract}
Emerging wireline transmission systems aspire to deliver rates in the order of Gbit/s over short copper cables by exploiting higher frequencies and non-differential transmission modes. Reliable channel models are a prerequisite for system design and throughput predictions. In this paper, a MATLAB-based multi-wire transmission line modeling toolbox called MTL is presented. Based on Kelvin-model theory, MTL allows modeling of virtually any property (spanning the entire range from electrical properties such as voltages, currents, and impedances to system-level properties such as insertion loss or Shannon capacity) of multi-wire cables with arbitrary wire geometry. Compared to prior art, MTL allows exact computation of the capacitance matrix in a multi-wire system. Examples demonstrate the importance of this feature.
\end{abstract}

Index Terms-Channel modeling, kelvin model, multi-conductor transmission line modeling, wireline communications.

\section{INTRODUCTION}

High-speed data transmission over copper wires is a key element of hybrid fiber-copper Internet access networks [1]. Exploiting high bandwidths and non-differential transmission modes, rates in the order Gbit/s can be achieved. Reliable channel models are the basis for system design. While the elementary theory of multi-conductor transmission line modeling is around hundred years old (cf. [2] and references therein), designers of emerging high-speed wireline communication systems face the lack of accurate channel models and supporting software. The goal of the tool presented in this paper is to bridge this gap. The multi-wire transmission line toolbox, hereinafter referred to as MTL, allows cable modeling for wireline communications based on the Kelvin model [2].

In agreement with the idea of reproducible research [3], a MATLAB implementation of MTL and all examples are available at http://mtlmodel.org and references to MTL functions are included in the following sections. Compared to previous work in the area-most notably [4]-MTL determines the exact solution for the capacitance matrix of the wire system solving a linear system of equations. Using exact capacitance results is particularly important for modeling alternative modes.

The paper is organized as follows. Section II briefly introduces the basics of the Kelvin modeling approach. Section III focuses on the capacitance solver. Section IV

Manuscript received October 25, 2012; revised November 29, 2012.

T. Magesacher is with the Department of Electrical and Information Technology, Lund University, P.O. Box 118, 22100 Lund, Sweden (e-mail: tom@eit.lth.se). presents application examples of the toolbox and Section V concludes the work.

\section{KELVIN MODELING}

The Kelvin model is the most elementary approach to capturing the properties of a multi-conductor transmission line system [5]. It is based on the classic electrical two-port model of an infinitely short multi-wire segment consisting of a serial impedance matrix $\boldsymbol{Z}=\boldsymbol{R}+j 2 \pi f \boldsymbol{L}$ and a shunt admittance matrix $\boldsymbol{Y}=\boldsymbol{G}+j 2 \pi f \boldsymbol{C}$. A segment is characterized exclusively by its geometry (wire center positions, wire diameters, etc.) and physical/electrical parameters (conductivity of conductor and insulation material, etc.). Cascading such segments, as illustrated in Fig. 1, multi-wire systems with arbitrary geometry can be modeled. The following elementary assumptions are made:

1) All conductors have circular cross-sections (and are thus referred to as wires).

2) All wires' cross-sections are small compared to the wavelength (which justifies the assumption of a transverse electromagnetic field structure surrounding the conductors).

3) Impact of wires' surface roughness or surface plating is neglected.

Simulation of a given scenario involves the following steps:

1) Division of wire-system into $N$ short segments (cf. Fig. 1) and setting up of wire geometry for each wire segment (fp3 and tp3 assist in computing space coordinates of flat pairs (or wires) and twisted pairs (or wires), respectively). tpsqueeze moves wire pairs closer together in an attempt to capture the effects of the "squeezing process" of pairs during cable assembly.

2) Computation of per-segment chain matrices $\boldsymbol{H}_{i}, i=1,2, \ldots, N$. The MTL functions rlcg and rlcg2chain yield primary matrix parameters and chain parameter matrix, respectively.

3) Computation of overall chain matrix $\boldsymbol{H}=\boldsymbol{H}_{N} \boldsymbol{H}_{N-1} \mathrm{~L} \boldsymbol{H}_{2} \boldsymbol{H}_{1}$.

4) Computation of characteristic impedance matrix $\boldsymbol{Z}_{\mathrm{c}}$ of wire system (using chain2zc).

5) Setting up of termination conditions for both source side and load side.

6) Solving for voltages $\boldsymbol{V}, \quad \boldsymbol{V}^{\prime}$ and currents $\boldsymbol{I}, \quad \boldsymbol{I}$ given the termination conditions (solveterm constructs and solves the linear system of equations).

7) Computation of parameters of interest (transfer paths, crosstalk paths, input impedances, etc.) 


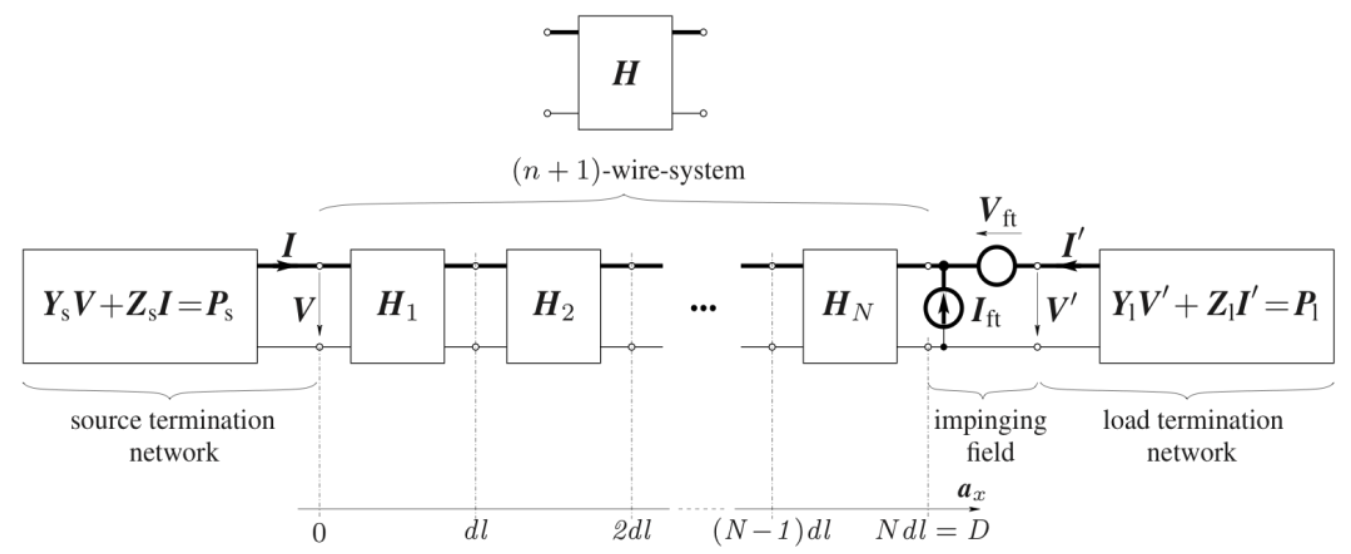

Fig. 1. System of $n+1$ wires with $N$ segments: segment No. $i$ is modeled by chain matrix $\boldsymbol{H}_{i}$. The entire wire system is modeled by $\boldsymbol{H}_{\text {. The }}$ source-side termination is described by $\boldsymbol{Y}_{\mathrm{S}} \boldsymbol{V}+\boldsymbol{Z}_{\mathrm{S}} \boldsymbol{I}=\boldsymbol{P}_{\mathrm{S}}$. The loadside termination is described by $\boldsymbol{Y}_{1} \boldsymbol{V}^{\prime}+\boldsymbol{Z}_{1} \boldsymbol{I}^{\prime}=\boldsymbol{P}_{1}$. The impact of an impinging electromagnetic field is modeled by equivalent concentrated sources $\boldsymbol{V}_{\mathrm{ft}}$ and $\boldsymbol{I}_{\mathrm{ft}}$. (For simplicity, hereinafter the transmission-line system is arranged along the $x$-axis. Note that MTL allows an arbitrary arrangement (for example, following a cable conduit or coiled up on a cable drum).).

In practice, exact wire-geometry information may not always be available. Nevertheless, MTL allows correct modeling of a multitude of properties for the given geometry information. The results can be adjusted to reality by introducing stochastic parameters.

\section{CAPacitance Solver}

Most models assume a uniform circumferential charge distribution on all wires. Let $\boldsymbol{L}_{\mathrm{e}}(k, m)$ denote a matrix containing mutual inductances $(k \neq m)$ and selfinductances $(k=m)$. The entries of $\boldsymbol{L}_{\mathrm{e}}(k, m)$ depend solely on wire geometry and medium properties and are given by

$$
\mathbf{L}_{\mathrm{e}}(k, m)=\left\{\begin{array}{ll}
\frac{\mu_{\mathrm{m}}}{2 \pi} \ln \frac{D_{k, 0}^{2}}{r_{0} r_{k}}, & k=m \\
\frac{\mu_{\mathrm{m}}}{2 \pi} \ln \frac{D_{k, 0} D_{m, 0}}{D_{k, m} r_{0}}, & \text { otherwise }
\end{array}, k, m \in\{1,2, \ldots, n\}\right.
$$

where $\mu_{\mathrm{m}}$ denotes the permeability of the medium, $r_{i}$ denotes the radius of wire No. $i, i=0,1, \ldots, n$ and $D_{i, j}$ is the distance between wire No. $i, i=0,1, \ldots, n$ and wire No. $j, j=0,1, \ldots, n, j \neq i$. The overall inductance matrix is given by

$$
\mathbf{L}(k, m)=\left\{\begin{array}{l}
\mathbf{L}_{\mathrm{e}}(k, m)+2 \ell(f), \quad k=m \\
\mathbf{L}_{\mathrm{e}}(k, m)+\ell(f), \quad \text { otherwise }
\end{array}, k, m \in\{1,2, \ldots, n\}\right.
$$

where $\ell(f)$ models frequency-dependent losses caused by imperfect conductor material often referred to as skin effect and proximity effect. Under the assumption that the medium is homogeneous, the capacitance matrix is given by

$$
\boldsymbol{C}=\mu_{\mathrm{m}} \varepsilon_{\mathrm{m}} \boldsymbol{L}_{\mathrm{e}}^{-1}
$$

where $\varepsilon_{\mathrm{m}}$ is the permittivity of the medium.
Computation of the inductance matrix according to (1) and subsequent computation of the capacitance matrix according to (3) implies two simplifying assumptions:

1) Neglecting the proximity effect

2) Assuming a homogeneous medium

In essence, Assumption 1 means that the circumferential charge distribution on each wire in the system is constant, which leads to regular geometry resulting in descriptions as simple as (1). However, wires in cables are typically squeezed closely together, which can result in significant deviation from the constant-charge-distribution assumption.

Assumption 2 implies that there is one and only one material surrounding the wires. For real cables, this is obviously never the case since wires have cylindrical insulation and the material in between conductors is thus a mixture of insulation material and air.

In order to drop these two assumptions, a capacitance-solver approach is required. The procedure can be summarized as follows (cf. [2] and references therein):

1) Compute the capacitance matrix $\boldsymbol{C}$ with inhomogeneous medium

2) Compute the capacitance matrix $\boldsymbol{C}_{0}$ assuming free space as medium

3) Assuming that the medium is not ferromagnetic, which holds for cables, the inductance matrix $\boldsymbol{L}_{\mathrm{e}}$ can be computed from $\boldsymbol{C}_{0}$ as

$$
\boldsymbol{L}_{\mathrm{e}}=\mu_{0} \boldsymbol{\varepsilon}_{0} \boldsymbol{C}_{0}^{-1}
$$

Equation (4) holds since inductance depends solely on the permeability of the surrounding medium and does not depend on the permittivity of the medium. The permeability of free space is $\mu_{0}$. Determining the exact capacitance solution involves three steps. First, the circumferential charge distribution on each wire is modeled using a Fourier series with $M_{1}$ coefficients per wire. Second, a system of equations is developed using two types of boundary conditions. Third, the system matrix is inverted in order to compute the generalized capacitance matrix. 
The boundary conditions are:

1) The potential of all points on conductor periphery is constant. Integration of charge distribution around the conductor periphery yields the contribution to the total potential.

2) The normal components of electric flux in points on the conductor/dielectric boundary are continuous. At the interface between insulation and air the electric field component tangent to the boundary must be continuous.

Mathematically, the boundary conditions can be formulated as

$$
\left[\begin{array}{l}
c \\
0
\end{array}\right]=A\left[\begin{array}{c}
a \\
a^{\prime}
\end{array}\right]
$$

where $c \in \mathbb{R}^{(n+1) M_{1} \times 1}$ denotes the conductor potentials, $\boldsymbol{a} \in \mathbb{R}^{(n+1) M_{1} \times 1}$ denotes the expansion coefficients of charge distribution on conductor and $\boldsymbol{a}^{\prime} \in \mathbb{R}^{(n+1) M_{1} \times 1}$ denotes the expansion coefficients of bound charge distribution at insulation/air interface. Inversion of the equation system yields

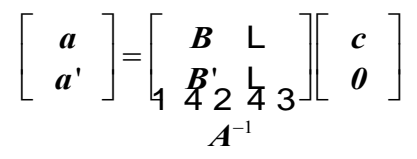

where $\boldsymbol{B}$ and $\boldsymbol{B}^{\prime}$ denote the sub-blocks of interest of the inverse $\boldsymbol{A}^{-1}$ of $\boldsymbol{A}$. Note that capacitance is charge divided by potential. Using the concept of generalized capacitance [2], which essentially eliminates the need for defining a reference conductor, the exact capacitance matrices $\boldsymbol{C}$ and $\boldsymbol{C}_{0}$ can thus be extracted from $\boldsymbol{B}$ and $\boldsymbol{B}^{\prime}$ without computing explicit solutions for charge distribution or potential (capsolve).
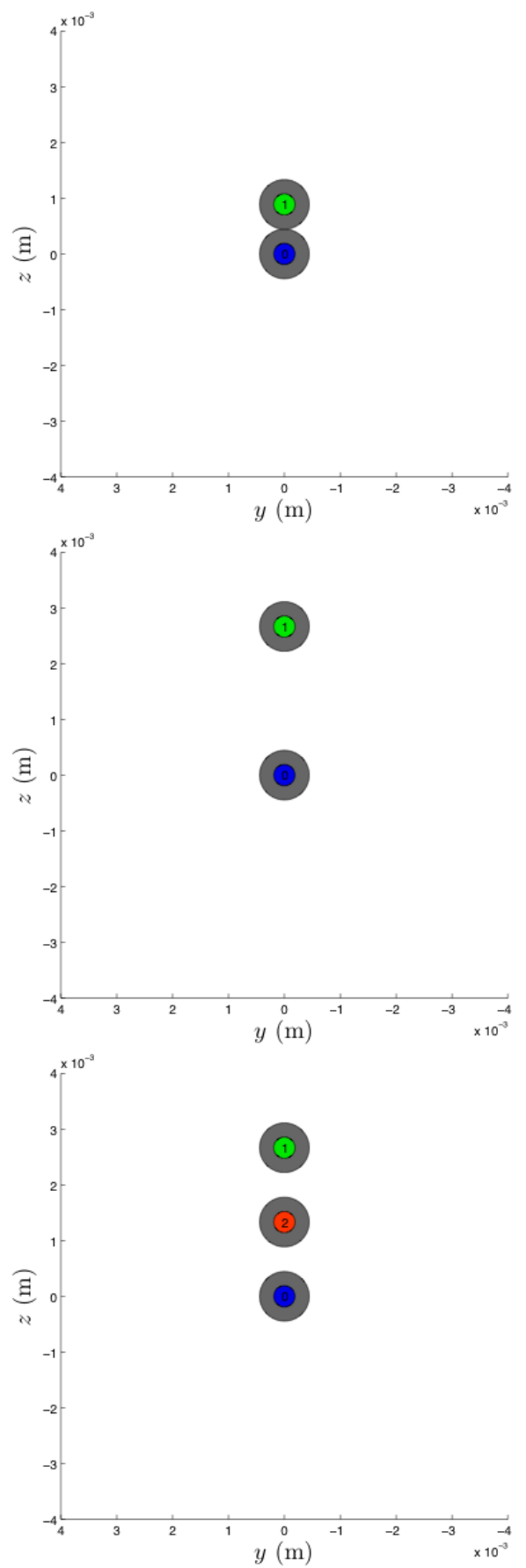
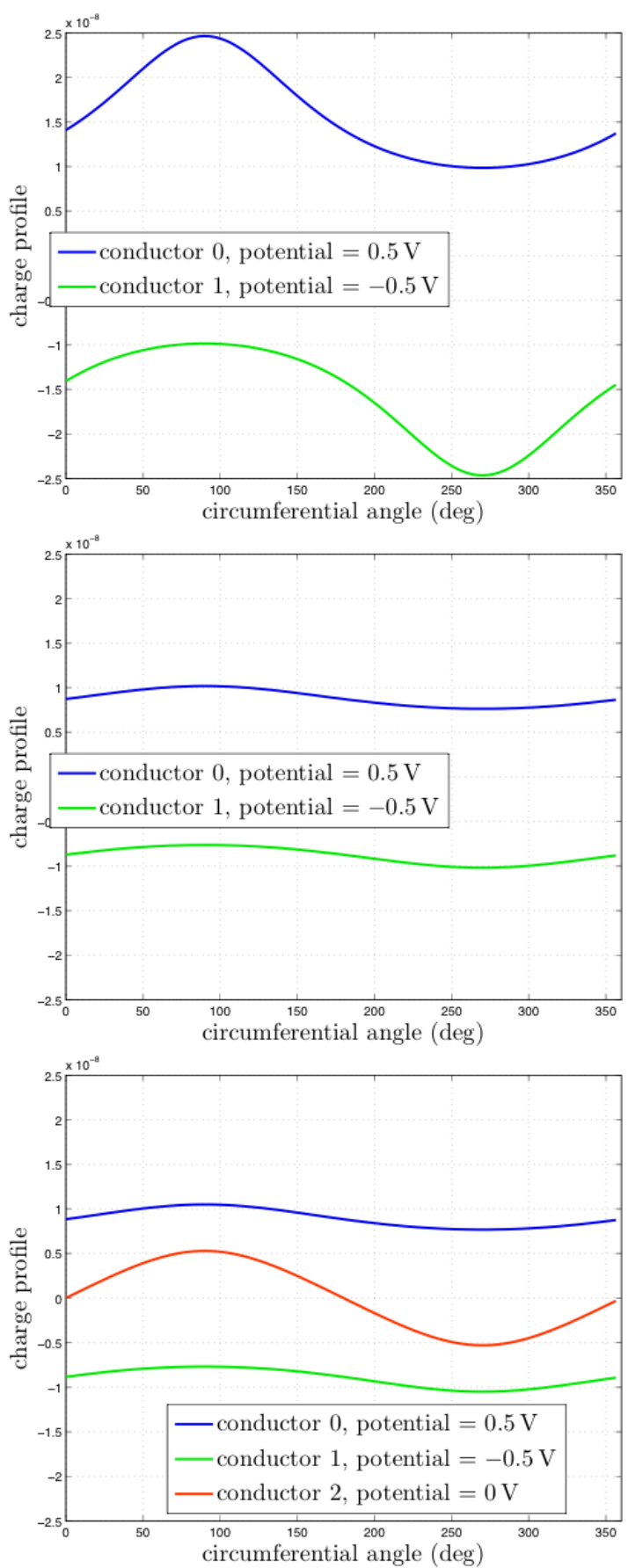

Fig. 2. (color) Cross-sections (left) and circumferential charge profiles (right) of example scenarios. 


\section{EXAMPLES}

In order to demonstrate the importance of exact capacitance information we consider three simple scenarios (cf. demo capacitance_solver_example). All copper wires have radius $r=0.191 \mathrm{~mm}$ and a cylindrical insulation of thickness $0.254 \mathrm{~mm}$ with permittivity $\varepsilon_{\mathrm{r}}=3.5$. All charge profiles are modeled using $M_{1}=21$ Fourier coefficients.

In order to compute charge distributions, the following potentials are applied to the wires: wire No. $0: 0.5 \mathrm{~V}$, wire No. 1: $-0.5 \mathrm{~V}$, wire No. $2: 0 \mathrm{~V}$.

\section{A. Scenario 1}

Two wires are spaced at minimum distance $\left(D_{1,0} / r=4.7\right)$ as shown in Fig. 2 (top, left). The approximate solution assumes a uniform charge distribution over circumference. With $\varepsilon_{\mathrm{m}}=\varepsilon_{0}$ (assuming that the surrounding medium is air), $\boldsymbol{C}_{11}=18.7 \mathrm{pF} / \mathrm{m}$. With $\varepsilon_{\mathrm{m}}=\varepsilon_{\mathrm{r}} \varepsilon_{0} \quad$ (assuming that the surrounding medium is insulation material), $C_{11}=65.3 \mathrm{pF} / \mathrm{m}$. The exact solution using the capacitance solver (and thus considering the mixture of insulation material and air in between the wires) is $C_{11}=41.5 \mathrm{pF} / \mathrm{m}$ and thus lies, not surprisingly, in between the values obtained for air and insulation. The charge distribution shown in Fig. 2 (top, right) deviates substantially from the uniform assumption.

\section{B. Scenario 2}

The two wires are now widely-spaced $\left(D_{1,0} / r=14\right)$, which decreases their mutual impact on charge distribution (cf. Fig. 2 (center, left)). However, the non-uniformity due to the proximity effect is still remarkable as shown in Fig. 2 (center, right). The capacitance results for $\boldsymbol{C}_{11}$ assuming $\varepsilon_{\mathrm{m}}=\varepsilon_{0} \quad$ and $\quad \varepsilon_{\mathrm{m}}=\varepsilon_{\mathrm{r}} \varepsilon_{0} \quad$ are $\quad 10.6 \mathrm{pF} / \mathrm{m}$ and $40 \mathrm{pF} / \mathrm{m}$, respectively. The exact solution computed by the capacitance solver is $\boldsymbol{C}_{11}=13.8 \mathrm{pF} / \mathrm{m}$, which is closer to the all-air approximation since the wire distance is large compared to wire diameters. For two wires, it might be possible to approximate $\boldsymbol{C}_{11}$ reasonably well by selecting a value that lies in between the two extreme values obtained for all-air and all-medium depending on the distance of the wires. However, this approach fails as soon as there is a third wire involved, as demonstrated in the next scenario.

\section{Scenario 3}

A third conductor is placed right in between conductors No. 0 and No. 1 while keeping the same distance between conductors No. 0 and No. 1 as in Scenario 2 (cf. Fig. 2 (bottom, left)). The third conductor strongly impacts the dielectric media inhomogeneity between conductors No. 0 and No. 1, which is reflected by the charge distribution shown in Fig. 2 (bottom, right). The exact solution for the capacitance $\boldsymbol{C}_{11}$ is $6.2 \mathrm{pF} / \mathrm{m}$, which lies outside the range $[10.6,40] \mathrm{pF} / \mathrm{m}$ defined by the extreme cases (all-air, all-medium).

\section{CONCLUSION}

Accurate modeling of multi-wire cables is a prerequisite for emerging wireline transmission systems. MTL is a toolbox for Kelvin-modeling, which is exclusively based on wire geometry and physical parameters. Determining the exact solution for the capacitance matrix, which is the distinguishing element of MTL compared to prior art, has an enormous impact on modeling results. The open-source tool MTL aspires to support ongoing and spark new research in the area of high-speed wireline communications.

\section{ACKNOWLEDGMENT}

This work was supported by the Swedish Research Council (VR) under grant No. 621-2008-4139 and by the Swedish Foundation for Strategic Research (SSF) under grant No. ICA08-0022.

\section{REFERENCES}

[1] P. Ödling, T. Magesacher, S. Höst, P. O. Börjesson, M. Berg, and E Areizaga, "The fourth generation broadband concept," IEEE Communications Magazine, vol. 47, no. 1, pp. 63-69, Jan. 2009.

[2] C. R. Paul, Analysis of Multiconductor Transmission Lines, ISBN 0-471-02080-X, Wiley, 1994.

[3] P. Vandewalle, J. Kovacevic, and M. Vetterli, "Reproducible research in signal processing - what, why, and how," IEEE Signal Processing Magazine, vol. 26, no. 3, pp. 37-47, May 2009.

[4] B. Lee, J. M. Cioffi, S. Jagannathan, K. Seong, Y. Kim, M. Mohseni, and M. H. Brady, "Binder MIMO channels," IEEE Transactions on Communications, vol. 55, no. 8, pp. 1617-1628, Aug. 2007.

[5] P. Boets and L. Van Biesen, "Metallic 2-wire parametric line models - a survey," in Proc. of XVIII IMEKO world congress, Metrology for Sustainable Development, Rio de Janeiro, Brazil, pp. 1-6, Sept. 2006.

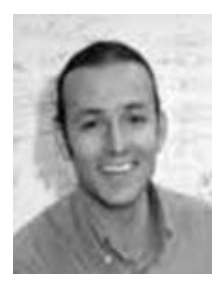

Thomas Magesacher received a Ph.D. degree in signal processing in 2006 and a Docent degree in telecommunications in 2011, both from Lund University, Sweden. He has five years of professional experience in the semiconductor industry working on the design and implementation of communication algorithms in Austria, Germany, and Israel. From 2000 till 2003, he worked at the Telecommunications Research Center Vienna (FTW), Austria, in the field of signal and information processing for communications. He spent the year 2007 as post-doctoral fellow in the group of Professor John M. Cioffi at the Electrical Engineering Department, Stanford University, USA. He has been involved in several startup ventures and is currently an Associate Professor at Lund University, Sweden. Dr. Magesacher was awarded an outgoing post-doctoral grant in 2006 and a four-year junior research grant in 2007 funding his assistant professor position, both from the Swedish Research Council. He received the Ingvar Carlsson Award from the Swedish Foundation for Strategic Research in 2009. His research interests include applied information processing, multi-conductor channel modeling, and applied optimization theory. 